\title{
Survival estimates and outcome predictors for shelter cats with feline panleukopenia virus infection
}

\author{
Federico Porporato DVM \\ Marian C. Horzinek† DVM, PhD \\ Regina Hofmann-Lehmann DVM \\ Filippo Ferri DVM \\ Gabriele Gerardi DVM \\ Barbara Contiero DStat \\ Tommaso Vezzosi DVM \\ Paola Rocchi DVM \\ Edoardo Auriemma DVM \\ Hans Lutz DVM, PhD \\ Eric Zini DVM, PhD
}

From the Istituto Veterinario di Novara, 28060 Granozzo con Monticello (NO), Italy (Porporato, Ferri, Vezzosi, Rocchi, Auriemma, Zini); former Institute of Virology, Department of Infectious Diseases and Immunology, Veterinary Faculty, Utrecht University, Yalelaan I, 3584 CL Utrecht, Netherlands (Horzinek); Clinical Laboratory, Vetsuisse Faculty, University of Zurich, 8057 Zurich, Switzerland (Hofmann-Lehmann, Lutz); Department of Animal Medicine, Production and Health, University of Padova, 35020 Legnaro (PD), Italy (Gerardi, Contiero, Zini); and Clinic for Small Animal Internal Medicine, Vetsuisse Faculty, University of Zurich, 8057 Zurich, Switzerland (Zini).

Address correspondence to Dr. Porporato (federico. porporato@istitutoveterinarionovara.it).

†Deceased.

\begin{abstract}
OBJECTIVE
To determine survival estimates and outcome predictors for shelter cats with feline panleukopenia virus (FPV) infection.

DESIGN

Retrospective cohort study.

\section{ANIMALS}

I77 shelter cats with FPV infection.

\section{PROCEDURES}

Medical records of cats treated for FPV infection from 2011 through 2013 were reviewed to collect information pertaining to signalment; history; results of physical examination, $C B C$, serum biochemical analysis, and blood gas analysis; and treatments (antimicrobials, antiparasitics, antivirals, antiemetics, analgesics, crystalloid or colloid solutions, and blood products). Survival time and outcome predictors were determined by means of $\mathrm{Ka}$ plan-Meier estimation, logistic regression, and mixed-model ANOVA.
\end{abstract}

\section{RESULTS}

Median survival time after hospital admission was 3 days; $20.3 \%$ (36/177) of cats survived to discharge from the hospital. Risk of nonsurvival was greater in cats with (vs without) signs of lethargy, rectal temperature $<37.9^{\circ} \mathrm{C}$ $\left(100.2^{\circ} \mathrm{F}\right)$, or low body weight at hospital admission. Lower (vs higher) leukocyte count on days 3,4 , and 7 of hospitalization, but not at admission, was associated with nonsurvival. Amoxicillin-clavulanic acid, antiparasitics, and maropitant but not interferon- $\omega$ were associated with survival, whereas glucose infusion was associated with nonsurvival.

\section{CONCLUSIONS AND CLINICAL RELEVANCE}

Results suggested that FPV infection carried a poor prognosis for shelter cats. Several variables measured at admission or during hospitalization were associated with outcome. Remarkably and contrary to the existing literature, leukopenia at admission had no association with outcome, possibly owing to early prevention of complications. (J Am Vet Med Assoc 2018;253:188-195)
$\mathbf{F}$ eline panleukopenia virus infection is an important infectious disease of cats that can also affect other mammalian species, including raccoons, foxes, minks, and ring-tailed cats. A nonenveloped, singlestranded DNA virus, FPV is a type of parvovirus that is genetically, structurally, and antigenically related to CPV, which can also infect cats (ie, CPV strains $2 a, 2 b$, and $2 c) .^{1-3}$ After a host is exposed, the virus replicates with high mitotic activity in tissues such as intestines, bone marrow, and, in young and adult cats, neurons. ${ }^{4,5}$ Replication in the bone marrow usually leads to immunosuppression caused by killing of myeloid colony progenitors. ${ }^{4}$

\section{ABBREVIATIONS}

$\begin{array}{ll}\mathrm{Cl} & \text { Confidence interval } \\ \mathrm{CPV} & \text { Canine parvovirus } \\ \text { FPV } & \text { Feline panleukopenia virus } \\ \text { RR } & \text { Relative risk }\end{array}$

Severity of FPV infection depends on the intensity of clinical signs on hospital admission and the degree of hematologic abnormalities (particularly of leukopenia) and can range from subclinical to peracute and deadly. ${ }^{6}$ The virus can easily spread from cat to cat in animal shelters and multicat households because of its strong environmental stability. Common clinical signs on initial evaluation of affected cats include fever, anorexia, dehydration, vomiting, and diarrhea. Diarrhea is less common than vomiting, ${ }^{7}$ and hemorrhagic diarrhea is uncommon. ${ }^{8}$ When death occurs, it is usually because of complications, including bacteremia or septicemia, severe dehydration, and disseminated intravascular coagulopathy. ${ }^{7}$ Infection with FPV has been associated with mortality rates of approximately $50 \%$ in cats., 10 Those surviving this infection for $>5$ days usually recover. ${ }^{8}$

Only 1 study ${ }^{10}$ has been reported in which factors associated with prognosis were investigated in cats with FPV infection. In that study, 244 cats re- 
ceived a diagnosis of FPV infection over a period of 17 years, and leukopenia, thrombocytopenia, hypoalbuminemia, and hypokalemia at hospital admission were associated with an increased risk of nonsurvival; median survival time was 2 days. ${ }^{10}$ Factors at admission were also examined for prognostic value, but no attempt was made to assess their role during the follow-up period and the effect of individual treatments was not investigated. In light of the paucity of existing data, the aim of the study reported here was to determine survival time and rate and identify factors at admission and during hospitalization that were associated with outcome for shelter cats with naturally acquired FPV infection.

\section{Materials and Methods}

\section{Cats}

An outbreak of FPV infection occurred in a large cat shelter located in the northwestern region of Italy at the end of autumn 2010 and persisted for $>3$ consecutive years, becoming endemic. Medical records of cats with FPV infection that were admitted to the veterinary hospital of the Istituto Veterinario di Novara, Granozzo con Monticello, Italy, between January 1, 2011, and December 31, 2013, were reviewed. Cats were included in the study if they had clinical or laboratory findings compatible with FPV infection and a positive result of fecal antigen ELISA testing for parvovirus $^{\mathrm{a}}$ at hospital admission, originated from the described animal shelter, were treated only at the hospital, and were monitored until recovery or death.

\section{Study design}

For all included cats, available data from diagnosis throughout the hospitalization period were reviewed, including information pertaining to signalment, history, physical examination, CBC, serum biochemical analysis, blood gas analysis, and treatments. Information was collected to the point of death or discharge from the hospital.

Despite the retrospective nature of the study, standardized procedures had been followed for every affected cat as routine at the study hospital. Specifically, during each day of hospitalization, physical examinations were performed every 12 hours, at approximately 7:00 AM and 7:00 PM, or more frequently if intensive care was required. Intravenous crystalloid fluid administration was performed for all cats according to individual needs and was discontinued when they were observed to voluntarily eat and no longer had fluid or electrolyte losses. In addition to crystalloid fluids, cats were treated with colloid solution, antimicrobials, antiparasitics, antiemetics, analgesics, anti-inflammatory agents, and electrolyte and vitamin supplements as well as blood derivatives on the basis of clinical and laboratory findings, in accordance with standard protocols. Intravenous glucose infusion was performed only for cats with blood glucose concentrations $\leq 60 \mathrm{mg} / \mathrm{dL}$. With regard to antiviral drugs, recombinant feline interferon- $\omega^{\mathrm{b}}(1 \mathrm{MU} / \mathrm{kg})$ was administered SC once a day for 3 consecutive days or less if the cat died before completing the treatment course. This treatment was administered to cats with FPV infection whenever available.

If blood sample collection was not deemed risky for a given cat (ie, the cat was not in critical condition), a CBC was performed on samples obtained on admission, prior to any treatment, and daily until death or discharge from the hospital. Similarly, when feasible, a full serum biochemical analysis was performed on admission, whereas during hospitalization, only selected analytes were remeasured, including total protein concentration, albumin concentration, and analytes considered relevant for treatment decisions (eg, potassium concentration). To achieve an adequate number of cats for followup analysis, data pertaining to hospitalization were retrieved at 24 hours ( 1 day), 48 hours (2 days), 72 hours ( 3 days), and 96 hours ( 4 days) as well as at 7 days, if cats remained alive and had not been discharged.

Blood samples measuring $\geq 1.2 \mathrm{~mL}$ were collected from a jugular vein on admission and placed into EDTA-containing tubes (CBC) and serum-separator tubes (serum biochemical analysis). To repeat these analyses during hospitalization, samples measuring 0.6 to $1.0 \mathrm{~mL}$ were collected. Analyses were performed by use of automated instruments ${ }^{\mathrm{c}, \mathrm{d}}$; when serum was not immediately processed, it was stored at $-20^{\circ} \mathrm{C}$ until processed. When the attending clinician deemed it necessary, an aliquot of blood was placed in a heparin-containing tube for immediate blood gas analysis.

\section{Statistical analysis}

Commercial software ${ }^{\mathrm{e}}$ was used for statistical analysis. Median overall survival time for all cats with FPV was calculated from the point of hospital admission by means of the Kaplan-Meier product-limit method. From the statistical standpoint, cats that remained alive at discharge from the hospital were censored and the percentage of these cats was calculated.

Variables recorded at hospital admission-Several variables were investigated for associations with survival to discharge from the hospital. Of the data collected at admission, the following signalment, history, and clinical variables were considered: age (< 12 months or $\geq 12$ months); sex (male or female); body weight; purebred status (yes or no); vaccination status (vaccinated or not against calicivirus, feline herpesvirus, and FPV infection); rectal temperature; heart rate; respiratory rate; systolic arterial blood pressure; signs of lethargy, dehydration, anorexia, vomiting, or diarrhea; and any respiratory sign (ie, sneezing, nasal discharge, or coughing). Complete blood count variables included leukocyte, erythrocyte, and platelet counts; Hct; erythrocyte mean corpuscular volume; and hemoglobin concentration. Serum biochemical variables included total protein, 
albumin, creatinine, urea, glucose, bilirubin, cholesterol, triglycerides, fructosamine, sodium, potassium, calcium, phosphate, and magnesium concentrations and alanine transaminase, aspartate transaminase, alkaline phosphatase, $\gamma$-glutamyltransferase, creatine kinase, and lactic dehydrogenase activities. Blood gas variables included $\mathrm{pH}, \mathrm{PCO}_{2}, \mathrm{PO}_{2}$, and bicarbonate and lactate concentrations. Furthermore, whether cats had positive or negative results of anti-FIV antibody and FeLV antigen testing ${ }^{\mathrm{f}}$ was also recorded. Variables that on univariate analysis (logistic regression) yielded a value of $P<0.05$ were retained to evaluate their independence by means of multivariate regression analysis. Relative risk and 95\% CIs were calculated. If any significant differences were identified for continuous variables, receiver operating characteristic curves were created to identify the best cutoff value for prediction of survival to discharge.

Variables recorded during hospitalization-Results of repeated CBCs and repeated measurements of serum total protein and albumin concentrations during hospitalization were assessed for associations with survival to hospital discharge by means of mixed-model ANOVA for repeated measurements, accounting for the effect of cat, survival status (ie, survivor or not), time, and their interaction. Among treatments provided during hospitalization, those tested to verify an association with survival on univariate analysis were antimicrobials (amoxicillin-clavulanic acid, ampicillin, cefazolin, ceftriaxone, and metronidazole), antiparasitics (milbemycin oxime-praziquantel, fenbendazole, or imidacloprid-moxidectin), recombinant feline interferon- $\omega$, antiemetics (maropitant and metoclopramide), NSAIDs (meloxicam), analgesics (buprenorphine and methadone), crystalloid solutions (lactated Ringer, saline $[0.9 \% \mathrm{NaCl}]$, and hetastarch solutions), IV administered supplements (glucose solution, potassium chloride, and multivitamin complex), any blood derivative, and feeding through nasal tube

Treatments that on univariate analysis yielded a value of $P<0.05$ were further analyzed for an association with survival by means of Cox proportional hazards modeling. For none of the listed treatments was an attempt made to investigate whether number of administrations was associated with outcome. Because treatment with recombinant feline interferon- $\omega$ was unevenly provided to cats, age, sex, body weight, and leukocyte count (recorded at admission) of those receiving or not the antiviral drug were compared by use of contingency tables and the Student $t$ test or Mann-Whitney $U$ test. Values of $P<0.05$ were considered significant for all analyses.

\section{Results}

\section{Cats}

Of the initial 265 cats with suspected FPV infection, 177 (66.8\%) met the inclusion criteria and were included in the study. Age was known for 133 of the $177(75.1 \%)$ cats, for which the median age was 3 months (range, 2 months to 3 years). Of the remaining $44(24.9 \%)$ cats with an unknown age, 36 were deemed older than 1 year (on the basis of dentition and body weight) and 8 younger than 1 year. Overall, $126(71.2 \%)$ cats were $<1$ year of age and 51 (28.8\%) cats were older. Eighty-four (47.5\%) cats were male and 93 (52.5\%) were female. Median body weight was $1 \mathrm{~kg}$ (2.2 lb; range, 0.27 to $6.70 \mathrm{~kg}$ [0.59 to $14.7 \mathrm{lb}]$ ). One hundred seventy-six (99.4\%) cats were domestic shorthairs, and 1 was a Birman.

Vaccination status was known for 142 (80.2\%) cats, of which only 2 (1.4\%) had been vaccinated. Both vaccinated cats were 3 months old and had received only 2 vaccines 4 weeks before hospital admission; this

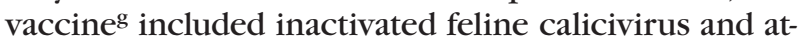
tenuated feline herpesvirus and FPV. Serologic FIV and FeLV testing ${ }^{\mathrm{f}}$ had been performed for 40 (22.6\%) cats, $4(10.0 \%)$ of which were seropositive for anti-FIV antibody and 2 (5.0\%) of which were seropositive for FeLV antigen. Of the 4 FIV-positive cats, 3 were adults and 1 was 1 month old; the positive result for the 1-monthold cat was deemed possibly attributable to maternally acquired antibody.

Major clinical signs, as assessed by veterinarians at the animal shelter, included gastrointestinal signs ( $n=88$ [49.7\%]), anorexia (86 [48.6\%]), lethargy (65 [36.7\%]), and respiratory signs (33 [18.6\%]). Specific gastrointestinal signs included both vomiting and diarrhea (35/88 [39.8\%]), vomiting only (27/88 [30.7\%]), diarrhea only (25/88 [28.4\%]), and hematochezia (1/88 [1.1\%]). Reported respiratory signs pertained to the upper respiratory tract (33/33 [100\%]) and lower respiratory tract (11/33 [33.3\%]). Physical examination findings at hospital admission included a median rectal temperature of $38.5^{\circ} \mathrm{C}\left(101.3^{\circ} \mathrm{F}\right.$; range, $33.0^{\circ}$ to $41.1^{\circ} \mathrm{C}$ [ $91.4^{\circ}$ to $106.0^{\circ} \mathrm{F}$ ), median heart rate of 200 beats/min (range, 80 to 250 beats $/ \mathrm{min}$ ), and median respiratory rate of 24 breaths/min (range, 8 to 60 breaths/min).

Thoracic radiography had been performed for 11 (33\%) cats in which concurrent lower respiratory tract disease was suspected. Pneumonia was identified in 6 of these cats and pleural effusion in 3, whereas findings for the remaining 2 cats were unremarkable.

\section{Survival estimates}

Median survival time after hospital admission for all cats with FPV infection was 3 days. One hundred forty-one $(79.7 \%)$ cats did not survive to discharge from the hospital, for an overall survival rate of $20.3 \%$ (36/177). Sixty-two (44.0\%) cats with a fatal outcome survived no longer than 2 days, 117 (83.0\%) died within 5 days, and all 141 (100\%) cats with a fatal outcome died within 15 days after hospital admission.

\section{Survival predictors at admission}

Of the evaluated signalment variables recorded at hospital admission, body weight was independently associated with survival, as determined through multivariate analysis. Indeed, for every $1-\mathrm{kg}$ increase in 
body weight, the chance of death decreased by $41 \%$ (RR, $0.59 ; 95 \% \mathrm{CI}, 0.39$ to $0.66 ; P=0.01$ ). The optimal cutoff value for differentiating survivors from nonsurvivors was $1.25 \mathrm{~kg}(2.75 \mathrm{lb})$, with a sensitivity of $69.6 \%$ and specificity of $64.6 \%$. No association with outcome was identified for cat age, sex, or purebred status on univariate analysis. Vaccination status was not included in the analysis owing to the small number of vaccinated cats.

Of the evaluated historical and clinical variables recorded at admission, signs of lethargy and rectal temperature were independently associated with outcome. Of the 65 cats with signs of lethargy, 59 (91\%) died during hospitalization; cats without these signs had approximately 6 times the chance of survival, compared with cats with these signs (RR, 6.05; $95 \% \mathrm{CI}, 1.73$ to $21.11 ; P=0.005)$. For each $1^{\circ} \mathrm{C}\left(1.8^{\circ} \mathrm{F}\right)$ increase in rectal temperature, the risk of death decreased by $32 \%$ (RR, $0.68 ; 95 \% \mathrm{CI}, 0.45$ to $1.02 ; P=$ $0.046)$. The receiver operating characteristic analysis for this variable indicated that the best cutoff value for differentiating survivors from nonsurvivors was $37.9^{\circ} \mathrm{C}\left(100.2^{\circ} \mathrm{F}\right)$, with a sensitivity of $100 \%$ and specificity of $39.6 \%$. No association with outcome was identified for heart rate, respiratory rate, and the clinical signs dehydration, anorexia, vomiting alone, diarrhea alone, or both vomiting and diarrhea on univariate analysis.

None of the evaluated $\mathrm{CBC}$, serum biochemical, or blood gas variables recorded at admission were associated with survival on univariate analysis. At admission, the prevalence of leukopenia $(<5$ $\times 10^{3}$ leukocytes $/ \mu \mathrm{L}$ ) was similar between cats that did (23/30 [77\%]) and did not (82/107 [77\%]) survive to discharge from the hospital, and leukocyte counts did not differ significantly between these 2 groups. Median number of leukocytes for survivors was $1.2 \times 10^{3} / \mu \mathrm{L}$ (range, $0.2 \times 10^{3} / \mu \mathrm{L}$ to $19.3 \times 10^{3} /$ $\mu \mathrm{L}$ ) and for nonsurvivors was $1.3 \times 10^{3} / \mu \mathrm{L}$ (range, 0 to $32.6 \times 10^{3} / \mu \mathrm{L}$ ). Cats with platelet aggregates reported in the $\mathrm{CBC}$ results were considered as missing values to avoid any underestimation. Of the 98 cats that had platelet counts without aggregates, thrombocytopenia $\left(<150 \times 10^{3}\right.$ platelets $\left./ \mu \mathrm{L}\right)$ was recorded for 11 of 20 (55\%) survivors (median, $125 \times 10^{3}$ platelets $/ \mu \mathrm{L}$; range, 0 to $398 \times 10^{3}$ platelets $/ \mu \mathrm{L})$ and 45 of $78(58 \%)$ nonsurvivors (121 X $10^{3}$ platelets $/ \mu \mathrm{L}$; range, 0 to $949 \times 10^{3}$ platelets/ $\mu \mathrm{L})$. Neither platelet counts nor proportions with thrombocytopenia differed between survivors and nonsurvivors.

Data on serum potassium concentration at hospital admission were available for 17 survivors (median, $3.8 \mathrm{mmol} / \mathrm{L}$; range, 3.0 to $5.6 \mathrm{mmol} / \mathrm{L}$ ) and 57 nonsurvivors (median, $4.3 \mathrm{mmol} / \mathrm{L}$; range, 3.0 to 5.8 mmol/L). Hypokalemia (potassium concentration < $3.3 \mathrm{mmol} / \mathrm{L})$ was recorded for 2 (12\%) survivors and 3 (5\%) nonsurvivors. Data on serum total protein concentration were available for 25 survivors (median, 68 $\mathrm{g} / \mathrm{L}$; range, 53 to $101 \mathrm{~g} / \mathrm{L}$ ) and 73 nonsurvivors (medi- an, $64 \mathrm{~g} / \mathrm{L}$; range, 13 to $106 \mathrm{~g} / \mathrm{L})$. Low concentrations $(<59 \mathrm{~g} / \mathrm{L})$ were recorded for 4 (16\%) survivors and 25 (34\%) nonsurvivors. Data on serum albumin concentration were available for 21 survivors (median, $28 \mathrm{~g} / \mathrm{L}$; range, 29 to $38 \mathrm{~g} / \mathrm{L}$ ) and 67 nonsurvivors (median, $26 \mathrm{~g} / \mathrm{L}$; range, 12 to $45 \mathrm{~g} / \mathrm{L}$ ). Hypoalbuminemia (albumin concentration $<27 \mathrm{~g} / \mathrm{L}$ ) was identified in 7 (33\%) survivors and 39 (58\%) nonsurvivors. None of these serum biochemical data differed significantly between the 2 groups.

Of the 2 FeLV-positive cats, one survived and the other died within 3 days of hospitalization, whereas 3 of the 4 FIV-positive cats died within a median of 9 days (range, 5 to 10 days).

\section{Survival predictors during hospitalization}

Results of multivariate analysis indicated that of the $\mathrm{CBC}$ variables measured during hospitalization, leukocyte count predicted survival. Indeed, survivors had significantly higher leukocyte counts than nonsurvivors at 72 hours $(P=0.001), 96$ hours $(P$ $=0.04)$, and 7 days $(P=0.004)$ of hospitalization (Figure I). Of note, with arbitrary reclassification of leukocyte data by severity of leukopenia, the RR of death remained fairly constant for each severity classification, whether at 72 hours, 96 hours, or 7 days of hospitalization (Table I). Leukocyte counts at 24 and 48 hours of hospitalization did not differ between survivors and nonsurvivors. None of the other evaluated $\mathrm{CBC}$ variables remeasured during hospitalization were associated with survival during hospitalization, nor were serum total protein or albumin concentrations.

With regard to treatments, amoxicillin-clavulanic acid, antiparasitic drugs, and maropitant were given during hospitalization to 30 (20.8\%), 21 (14.6\%),

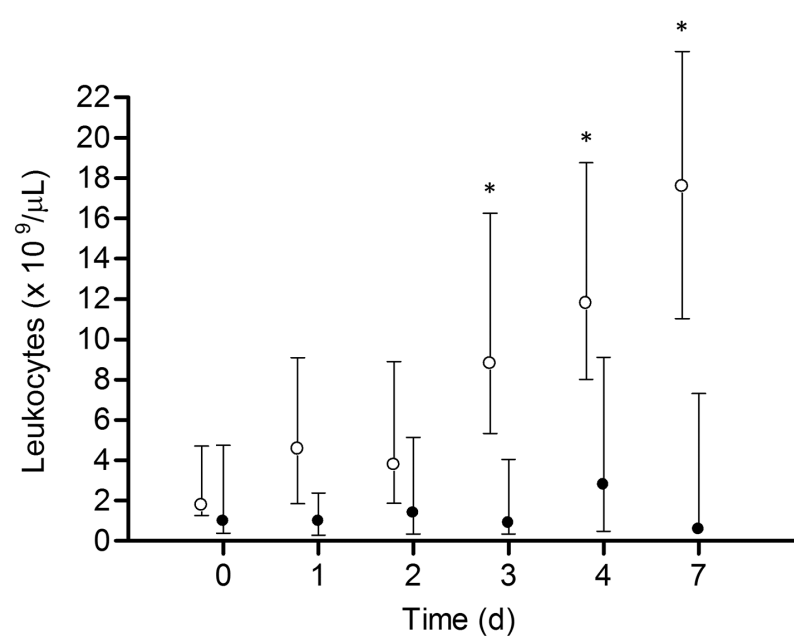

Figure I-Median leukocyte counts at various points during hospitalization for shelter cats with FPV infection that did $(n=36$; white circles) or did not (14I; black circles) survive to discharge from the hospital. Error bars represent interquartile range. Data points for the 2 groups are offset to allow distinction. *Median value differs significantly $(P<0.05)$ between groups. 
Table I-Estimated RRs of nonsurvival for I77 cats with FPV infection and various degrees of leukopenia at 72 hours, 96 hours, and 7 days of hospitalization.

\begin{tabular}{|c|c|c|c|c|c|c|c|c|c|}
\hline \multirow[b]{2}{*}{$\begin{array}{l}\text { Leukocyte count } \\
\left.\text { (X } 10^{3} / \mu \mathrm{L}\right)\end{array}$} & \multicolumn{3}{|c|}{72 hours } & \multicolumn{3}{|c|}{96 hours } & \multicolumn{3}{|c|}{7 days } \\
\hline & $\begin{array}{l}\text { No. of } \\
\text { cats }\end{array}$ & $\mathbf{R R}$ & $P$ value & $\begin{array}{l}\text { No. of } \\
\text { cats }\end{array}$ & $\mathbf{R R}$ & $P$ value & $\begin{array}{l}\text { No. of } \\
\text { cats }\end{array}$ & $\mathbf{R R}$ & $P$ value \\
\hline$<5$ & 26 & 2.27 & 0.001 & 9 & 1.80 & 0.04 & 8 & 3.64 & 0.004 \\
\hline$<4$ & 26 & 2.43 & $<0.001$ & 8 & 2.14 & 0.007 & 8 & 3.64 & 0.004 \\
\hline$<3$ & 24 & 2.39 & $<0.001$ & 8 & 2.00 & 0.01 & 8 & 4.20 & 0.001 \\
\hline$<2$ & 23 & 2.57 & $<0.001$ & 8 & 2.00 & 0.01 & 8 & 4.20 & 0.001 \\
\hline$<1$ & 18 & 2.33 & $<0.001$ & 6 & 1.80 & 0.045 & 7 & 3.42 & 0.004 \\
\hline
\end{tabular}

Values for number of cats represent the total number of all cats with the indicated leukocyte count.

and $30(20.8 \%)$ cats $(\mathrm{n}=144)$, respectively. Use (vs no use) of amoxicillin-clavulanic acid was associated with a $69 \%$ decrease in the risk of nonsurvival (RR, $0.31 ; 95 \% \mathrm{CI}, 0.09$ to $1.00 ; P=0.049)$, use of antiparasitic drugs was associated with an $87 \%$ decrease $(\mathrm{RR}$, $0.13 ; 95 \% \mathrm{CI}, 0.03$ to $0.50 ; P<0.001$ ), and use of maropitant was associated with an $84 \%$ decrease $(\mathrm{RR}$, $0.16 ; 95 \% \mathrm{CI}, 0.04$ to $0.70 ; P=0.01$ ). Furthermore, IV glucose infusion was performed for 65 of 144 (45.1\%) cats during hospitalization, and such treatment (vs no such treatment) was associated with an 11-fold increase in the risk of death (RR, 11.0; 95\% CI, 2.5 to $32.7 ; P<0.001$ ). No association with outcome was identified on univariate analysis regarding use of ampicillin, cefazolin, ceftriaxone, metronidazole, recombinant feline interferon- $\omega$, metoclopramide, meloxicam, buprenorphine or methadone, lactated Ringer or saline solution, hetastarch solution, potassium chloride, multivitamin complex, blood derivatives, or nasal-tube feeding.

Because only 70 (39.5\%) cats received feline recombinant interferon- $\omega$ during hospitalization, additional analyses were performed to verify whether population characteristics of those that received this antiviral were similar to those that did not receive it. Results indicated that at admission, sex and age distribution as well as body weight and leukocyte counts did not differ significantly between these 2 groups.

\section{Discussion}

In the present study involving mainly young cats and unvaccinated kittens from an animal shelter, FPV infection had a poor outcome, and cats without signs of lethargy, with a higher body weight, or with a higher rectal temperature at hospital admission were more likely to survive than other cats. Moreover, leukopenia on or after the third day of hospitalization was associated with a poor outcome. Treatment with amoxicillin-clavulanic acid, antiparasitics, or maropitant was associated with survival to discharge from the hospital, and IV glucose infusion was associated with nonsurvival.

Similar to findings in a previous study,$^{10}$ cats with FPV infection had a poor prognosis, with a briefer life expectancy (median, 3 days) than those without FPV infection. However, the survival rate in the present study was only $20.3 \%$, compared with $51 \%$ in the other study. ${ }^{10}$ The reason for this difference remains unclear, given that no association has been found between living conditions and survival rate..$^{10}$

The finding that cats with FPV infection were more likely to survive if they had a higher (vs lower) body weight at admission was not surprising. In general, thinness or even cachexia indicates that the amount of body substrates available to meet energy requirements is reduced; consequently, thin cats could be expected to die earlier than heavier cats if they had a severe infection such as that caused by FPV. Indeed, 2 previous studies ${ }^{11,12}$ involving dogs with parvovirus infection yielded results comparable to ours. In particular, one study ${ }^{11}$ showed that for each kilogram increase in body weight, the mortality rate of affected dogs decreased by $73 \%$, and in the other study, ${ }^{12}$ dogs that died had a lower body weight than those that survived. In the present study, body weight was used instead of body condition score, for which no data were unavailable; however, body condition score might have provided additional insights into the role of lean and fat mass in cats with FPV infection.

Regarding the observed association between high rectal temperature and survival for the cats of the present study, rectal temperature of healthy kittens after 3 weeks of age has been shown to increase linearly. ${ }^{13}$ In addition, for kittens $>7$ weeks of age, the lower reference limit for rectal temperature is $38.1^{\circ} \mathrm{C}$ $\left(100.6^{\circ} \mathrm{F}\right)$, which is less than the lower reference limit for adults. ${ }^{13}$ In the present study, in which almost half of included cats were kittens, the best discriminating cutoff value for predicting which cats at hospital admission were less likely to survive was $37.9^{\circ} \mathrm{C}$. Overall, we believe our results confirm that even a mild degree of hypothermia should be carefully regarded in young cats with FPV infection, suggesting that an advanced stage of disease and potential for nonsurvival may exist. Likewise, signs of lethargy also predicted a worse outcome, probably reflecting a more critical condition. Similar results have been reported for dogs with parvovirosis. ${ }^{14}$

With regard to $\mathrm{CBC}$ variables, unexpectedly and differently from previously reported findings, ${ }^{10}$ leukopenia at hospital admission had no association with nonsurvival for cats with FPV infection. To the authors' knowledge, no studies have been reported regarding the role of leukocyte counts throughout 
the hospitalization period in cats, only in dogs. ${ }^{15}$ The present study showed that cats with leukopenia $\geq 72$ hours after admission had an increased risk of death, compared with the risk for cats without leukopenia. In general, the degree of leukopenia during FPV or $\mathrm{CPV}$ infection is believed to reflect the severity of the disease, suggesting that patients with more severe leukopenia are in poorer health status and, hence, have a poorer prognosis. ${ }^{16}$ However, initial leukocyte counts and percentages of cats with leukopenia were similar between surviving and nonsurviving cats in the present study.

The finding that low leukocyte count in cats with FPV infection predicted outcome but only after 3 days of hospitalization might have suggested that prolonged leukopenia is associated with profound immunosuppression, which increases the chance of secondary infections and, in turn, the risk of death. Interestingly, irrespective of the degree of leukopenia at 72 hours, 96 hours, or 7 days of hospitalization, the RR of death was fairly constant; for instance, at 72 hours, cats with $<1 \times 10^{3}$ leukocytes/ $\mu \mathrm{L}$ had a chance of nonsurvival similar to that for cats with < $4 \times 10^{3}$ leukocytes $/ \mu \mathrm{L}$. Conversely, the RR for cats at 7 days of hospitalization was almost double that at 72 hours. Collectively, these findings suggested that the persistence rather than degree of leukopenia was associated with a poor prognosis in cats with FPV infection.

Contrary to previously reported findings for cats and dogs with parvovirosis ${ }^{10,17}$ hypoalbuminemia at hospital admission had no association with outcome in the present study. One possible reason for this difference could have been that the degree of hypoalbuminemia was not marked in most affected cats, and severe hypoalbuminemia may indicate a poor prognosis for some diseases in cats. ${ }^{18,19}$ Indeed, although half of the cats had a low serum albumin concentration at hospital admission, only approximately a fourth of them (data not shown) had severe hypoalbuminemia $(<20 \mathrm{~g}$ of albumin/L). Furthermore, hypokalemia and thrombocytopenia at hospital admission had no association with outcome, despite previously reported findings that low serum potassium concentration and low platelet count predict a poor outcome for cats with FPV infection. ${ }^{10}$ Of note, for dogs with parvovirosis, hypokalemia and thrombocytopenia are not associated with survival. ${ }^{20,21}$

With regard to treatments provided during hospitalization, the present study showed that treatment with amoxicillin-clavulanic acid was associated with an increased likelihood of survival for cats with FPV infection. Because replication of the parvovirus within intestinal cells enhances permeability of the intestinal mucosa and favors translocation of bacteria (particularly gram-negative and anaerobic bacteria) into circulation, ${ }^{8,22}$ use of a broad-spectrum antimicrobial can be presumed suitable to protect cats with FPV infection from this effect and thereby improve their chance of survival. This antimicrobial combination had been used because of its broad-spectrum activity against gram-positive, gram-negative, and anaerobic bacteria. ${ }^{23}$ It is worth noting that the other antimicrobials administered to cats in the present study (ie, ampicillin, cefazolin, ceftriaxone, and metronidazole) were not as beneficial, and their narrower spectrum of activity relative to amoxicillin-clavulanic acid could explain this limited success.

In the present study, antiparasitic treatment was associated with an increased chance of survival. Previous studies ${ }^{24-26}$ have shown that intestinal parasitism is common in shelter cats, particularly those younger than 6 months, and diarrhea may or not be a result. Moreover, shelters are potentially stressful environments owing to high group density, and stress may increase susceptibility to infections. ${ }^{27,28} \mathrm{Fecal}$ examination was not routinely performed when the cats were admitted to the study hospital because fecal samples were not available at that time or most cats had no diarrhea. However, considering that the cats originated from a shelter and were quite young, we surmise that several of them likely harbored enteric parasites and deworming would therefore have been useful. Several types of antiparasitics had been administered, but because few cats (14.6\%) received antiparasitic treatment, no analysis could be performed to assess whether certain products were more beneficial than others.

Maropitant had been administered to cats in the present study to prevent vomiting and dehydration caused by FPV infection, and this treatment was associated with survival. On the other hand, administration of metoclopramide had no such association. These results may be explained by the fact that maropitant has a greater antiemetic effect than metoclopramide, as shown in dogs. ${ }^{29}$ Indeed, maropitant acts centrally as a neurokin-1 receptor antagonist, whereas metoclopramide acts as a dopamine receptor inhibitor in the chemoreceptor trigger zone. However, dopamine receptors are lacking in cats, reducing the antiemetic effects of metoclopramide in this species. ${ }^{30}$

Unexpectedly, IV glucose infusion (vs no infusion) was strongly associated with a lower likelihood of survival for cats in the present study. In the authors' opinion, the reason behind this finding is not that glucose infusion was detrimental, but rather that cats received the infusion when in critical condition due to overwhelming sepsis. In this scenario, cats would be expected to have a lower chance of survival. Although blood glucose measurements had routinely been performed with a portable glucose meter at the study hospital during emergencies, few of these measurements were retrieved from medical records and so additional information regarding the severity of hypoglycemia could not be provided.

Of note, administration of recombinant feline interferon- $\omega$ was not associated with an increased chance of survival for cats with FPV infection in the present study. This finding was unexpected given its reported beneficial effects in feline fetus cells in vitro, in which the yield of FPV decreases with cell ex- 
posure to this antiviral drug, ${ }^{31}$ or in dogs with experimentally induced CPV infection, in which treatment is associated with improvement of clinical signs and a lower chance of nonsurvival. ${ }^{32}$ The preventive use of recombinant feline interferon- $\omega$ in a multicat household was shown to increase the amount of anti-FPV antibody in infected kittens that survive, although such treatment has no effect on the time of emergence of clinical signs or survival rate. ${ }^{33}$ No studies have been performed to assess the effect of recombinant feline interferon- $\omega$ in cats with established FPV infection.

The present study had some limitations that warrant discussion. In particular, owing to the retrospective nature of the investigation, some medical records were incomplete. In addition, some serum biochemical variables, such as albumin and potassium concentrations, during hospitalization could not be investigated to assess their association with outcome because of the limited number of cats with available data during the follow-up period. However, it is worth mentioning that nearly half of the cats were kittens, which would have hindered repeated collection of large blood samples, and so this limitation would have existed in a prospective study as well.

Regardless of any limitations, the shelter cats with FPV infection in the present study had a poor prognosis. Several variables were identified that could help clinicians identify cats at higher risk of nonsurvival than others and assist in patient management.

\section{Acknowledgments}

The study was performed at the Istituto Veterinario di Novara, Granozzo con Monticello, Italy.

The authors declare that there were no conflicts of interest.

Presented in abstract form at the 25th Annual Congress of the European College of Veterinary Internal Medicine, Lisbon, Portugal, September 2015.

The authors thank Drs. Laura Zamperone, Carmelo Parisi, Davide Pozzi, and Daniela Monfroglio for providing care to the shelter cats.

\section{Footnotes}

a. SNAP parvo test, Idexx Laboratories, Milan, Italy.

b. Virbagen omega, Virbac, Carros Cedex, France

c. Sysmex XT-2000iV, Sysmex Corp, Kobe, Japan.

d. P-modular 800, Hitachi Ltd, Tokyo, Japan.

e. SAS, version 9.3, SAS Institute Inc, Cary, NC.

f. SNAP FIV/FeLV combo plus test, Idexx Laboratories, Milan, Italy.

g. Purevax RCP, Merial, Lyon, France.

\section{References}

1. Mochizuki M, Harasawa R, Nakatani H. Antigenic and genomic variabilities among recently-prevalent parvoviruses of canine and feline origin in Japan. Vet Microbiol 1993;38:1-10.

2. Mochizuki M, Horiuchi M, Hiragi $\mathrm{H}$, et al. Isolation of canine parvovirus from a cat manifesting clinical signs of feline panleukopenia. J Clin Microbiol 1996;34:2101-2105.

3. Miranda C, Parrish CR, Thompson G. Canine parvovirus 2c infection in a cat with severe clinical disease. J Vet Diagn Invest 2014;26:462-464.

4. Parrish CR. Pathogenesis of feline panleukopenia virus and canine parvovirus. Baillieres Clin Haematol 1995;8:57-71.
5. Garigliany M, Gilliaux G, Jolly S, et al. Feline panleukopenia virus in cerebral neurons of young and adult cats. BMC Vet Res 2016;12:28.

6. Clegg SR, Coyne KP, Dawson S, et al. Canine parvovirus in asymptomatic feline carriers. Vet Microbiol 2012;157:78-85.

7. Addie DD, Jarrett O, Simpson J, et al. Feline parvovirus in pedigree kittens. Vet Rec 1996;138:119.

8. Greene CE. Feline enteric viral infections. In: Green CE, ed. Infectious diseases of the dog and cat. St Louis: Elsevier Saunders, 2012;80-91.

9. Truyen U, Addie D, Belăk S, et al. Feline panleukopenia: ABCD guidelines on prevention and management. J Feline Med Surg 2009;11:538-546.

10. Kruse BD, Unterer S, Horlacher K, et al. Prognostic factors in cats with feline panleukopenia. $J$ Vet Intern Med 2010;24:1271-1276.

11. Dossin O, Rupassara SI, Weng HY, et al. Effect of parvoviral enteritis on plasma citrulline concentration in dogs. J Vet Intern Med 2011;25:215-221

12. Schoeman JP, Herrtage ME. Serum thyrotropin, thyroxine and free thyroxine concentrations as predictors of mortality in critically ill puppies with parvovirus infection: a model for human paediatric critical illness? Microbes Infect 2008;10:203-207.

13. Mähler M, Riedesel H, Gärtner K, et al. Physiologic hypothermia of newborn kittens up to 6 weeks old. Zentralbl Veterinarmed A 1993;40:393-396.

14. Miranda C, Carvalheira J, Parrish CR, et al. Factors affecting the occurrence of canine parvovirus in dogs. Vet Microbiol 2015;180:59-64

15. Goddard A, Leisewitz AL, Christopher MM, et al. Prognostic usefulness of blood leukocyte changes in canine parvoviral enteritis. J Vet Intern Med 2008;22:309-316.

16. Cohn LA, Rewerts JM, McCaw D, et al. Plasma granulocyte colony-stimulating factor concentrations in neutropenic, parvoviral enteritis-infected puppies. J Vet Intern Med 1999;13:581-586.

17. Allenspach K, Wieland B, Gröne A, et al. Chronic enteropathies in dogs: evaluation of risk factors for negative outcome. J Vet Intern Med 2007;21:700-708.

18. Pyle SC, Marks SL, Kass PH. Evaluation of complications and prognostic factors associated with administration of total parenteral nutrition in cats: 75 cases (1994-2001). J Am Vet Med Assoc 2004;225:242-250.

19. Declue AE, Delgado C, Chang $\mathrm{CH}$, et al. Clinical and immunologic assessment of sepsis and the systemic inflammatory response syndrome in cats. J Am Vet Med Assoc 2011;238:890-897.

20. Mann FA, Boon GD, Wagner-Mann CC, et al. Ionized and total magnesium concentrations in blood from dogs with naturally acquired parvoviral enteritis. J Am Vet Med Assoc 1998;212:1398-1401.

21. Yilmaz Z, Senturk S. Characterisation of lipid profiles in dogs with parvoviral enteritis. J Small Anim Pract 2007;48:643-650.

22. Lamm CG, Rezabek GB. Parvovirus infection in domestic companion animals. Vet Clin North Am Small Anim Pract 2008;38:837-850.

23. Green CE, Calpin J. Appendix: antimicrobial drug formulary. In: Green CE, ed. Infectious diseases of the dog and cat. St Louis: Elsevier Saunders, 2012;1207-1320.

24. Hill SL, Cheney JM, Taton-Allen GF, et al. Prevalence of enteric zoonotic organisms in cats. I Am Vet Med Assoc 2000;216:687-692

25. Paris JK, Wills S, Balzer HJ, et al. Enteropathogen co-infection in UK cats with diarrhoea. BMC Vet Res 2014;10:13.

26. Sabshin SJ, Levy JK, Tupler T, et al. Enteropathogens identified in cats entering a Florida animal shelter with normal feces or diarrhea. J Am Vet Med Assoc 2012;241:331-337.

27. Kessler MR, Turner DC. Effects of density and cage size on stress in domestic cats (Felis sylvestris catus) housed in animal shelters and boarding catteries. Anim Welf 1999;8:259-267.

28. Buffington CA. External and internal influences on disease risk in cats. J Am Vet Med Assoc 2002;220:994-1002.

29. Sedlacek HS, Ramsey DS, Boucher JF, et al. Comparative efficacy of maropitant and selected drugs in preventing emesis 
induced by centrally or peripherally acting emetogens in dogs. J Vet Pharmacol Ther 2008;31:533-537.

30. King GL. Animal models in the study of vomiting. Can J Physiol Pharmacol 1990;68:260-268.

31. Mochizuki M, Nakatani H, Yoshida M. Inhibitory effects of recombinant feline interferon on the replication of feline enteropathogenic viruses in vitro. Vet Microbiol 1994;39:145152.
32. Martin V, Najbar W, Gueguen S, et al. Treatment of canine parvoviral enteritis with interferon-omega in a placebo-controlled challenge trial. Vet Microbiol 2002;89:115-127.

33. Paltrinieri S, Crippa A, Comerio T, et al. Evaluation of inflammation and immunity in cats with spontaneous parvovirus infection: consequences of recombinant feline interferon-omega administration. Vet Immunol Immunopathol 2007;118:68-74.

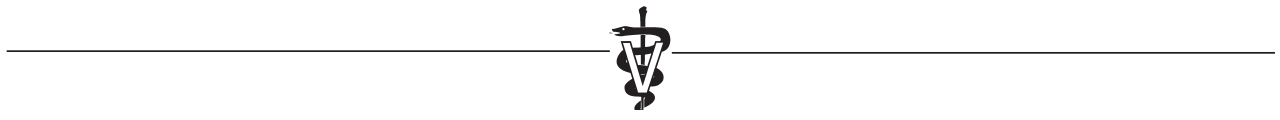

\section{From this month's AJVR}

\section{Hemodynamic effects of MK-467 following intravenous administration to isoflurane-anesthetized cats concurrently receiving dexmedetomidine}

Manuel Martin-Flores et al

\section{OBJECTIVE}

To evaluate the efficacy of each of 3 incremental doses of MK-467 for alleviation of dexmedetomidine-induced hemodynamic depression in isoflurane-anesthetized cats.

\section{ANIMALS}

6 healthy adult domestic shorthair cats.

\section{PROCEDURES}

Each cat was anesthetized with isoflurane and received a target-controlled infusion of dexmedetomidine estimated to maintain the plasma dexmedetomidine concentration at $10 \mathrm{ng} / \mathrm{mL}$ throughout the experiment. Heart rate (HR) and direct arterial pressures were measured at baseline (isoflurane administration only), during dexmedetomidine infusion, and before and after IV administration of each of 3 serially increasing doses $(15,30$, and $60 \mu \mathrm{g} / \mathrm{kg}$ ) of MK-467. Cardiac index (Cl) and systemic vascular resistance (SVR) were recorded at baseline, during dexmedetomidine infusion, and at the mean arterial pressure nadir after administration of the 30 - and $60-\mu \mathrm{g} / \mathrm{kg}$ doses of MK-467.

\section{RESULTS}

Compared with baseline values, the dexmedetomidine infusion significantly decreased HR and increased arterial pressures. Each dose of MK-467 caused a significant decrease in arterial pressures and a significant, albeit clinically irrelevant, increase in $\mathrm{HR}(\leq 10 \%)$. Following administration of the 30 - and $60-\mu \mathrm{g} / \mathrm{kg}$ doses of MK-467, all cats developed clinical hypotension (mean arterial pressure, $<60 \mathrm{~mm} \mathrm{Hg}$ ) even though $\mathrm{Cl}$ and SVR returned to baseline values.

\section{CONCLUSIONS AND CLINICAL RELEVANCE}

Results indicated administration of small doses of MK-467 to isoflurane-anesthetized cats receiving dexmedetomidine restored $\mathrm{Cl}$ and SVR, but caused a substantial decrease in arterial pressures and only a marginal increase in HR. Therefore, caution should be used when MK- 467 is administered to alleviate dexmedetomidine-induced hemodynamic depression in isoflurane-anesthetized cats. (Am J Vet Res 2018;79:71I-717)

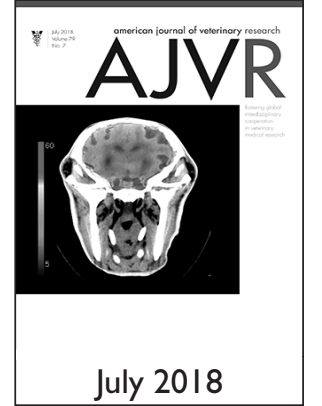

See the midmonth

issues of JAVMA

for the expanded

table of contents

for the AJVR

or log on to

avmajournals.avma.org

for access

to all the abstracts. 